\title{
On the convergence of iteration processes for semigroups of nonlinear mappings in modular function spaces
}

\author{
Buthinah A Bin Dehaish ${ }^{1 *}$, Mohamed A Khamsi ${ }^{2,3}$ and Wojciech M Kozlowski ${ }^{4}$
}

\section{"Correspondence:}

bbendehaish@kau.edu.sa; bbindehaish@yahoo.com

'Department of Mathematics, King

Abdulaziz University, P.O. Box 53909,

Jeddah, 21593, Saudi Arabia

Full list of author information is

available at the end of the article

\begin{abstract}
Let $C$ be a $\rho$-bounded, $\rho$-closed, convex subset of a modular function space $L_{\rho}$. We investigate the problem of constructing common fixed points for asymptotic pointwise nonexpansive semigroups of mappings $T_{t}: C \rightarrow C$, i.e. a family such that $T_{0}(f)=f, T_{s+t}(f)=T_{s} \circ T_{t}(f)$, and $\rho(T(f)-T(g)) \leq \alpha_{t}(f) \rho(f-g)$, where $\limsup _{t \rightarrow \infty} \alpha_{t}(f) \leq 1$, for every $f \in C$.

MSC: Primary 47H09; secondary 46B20; 47H10; 47E10

Keywords: fixed point; modular function space; nonexpansive mapping; Orlicz space; pointwise Lipschitzian mapping; pointwise nonexpansive mapping; semigroup; uniform convexity
\end{abstract}

\section{Introduction}

In 2008, Kirk and Xu [1] studied existence of fixed points of asymptotic pointwise nonexpansive mappings $T: C \rightarrow C$, i.e.

$$
\left\|T^{n}(x)-T^{n}(y)\right\| \leq \alpha_{n}(x)\|x-y\|
$$

where $\lim \sup _{n \rightarrow \infty} \alpha_{n}(x) \leq 1$, for all $x, y \in C$. Their main result (Theorem 3.5) states that every asymptotic pointwise nonexpansive self-mapping of a nonempty, closed, bounded and convex subset $C$ of a uniformly convex Banach space $X$ has a fixed point. As pointed out by Kirk and $\mathrm{Xu}$, asymptotic pointwise mappings seem to be a natural generalization of nonexpansive mappings. The conditions on $\alpha_{n}$ can be for instance expressed in terms of the derivatives of iterations of $T$ for differentiable $T$. In 2009, these results were generalized by Hussain and Khamsi to metric spaces [2]. In 2011, Khamsi and Kozlowski [3] extended their result proving the existence of fixed points of asymptotic pointwise $\rho$-nonexpansive mappings acting in modular function spaces. The existence of common fixed points of semigroups of nonexpansive (in a modular sense) mappings acting in modular function spaces was first established by Kozlowski in [4] and then extended to the semigroups of asymptotic pointwise nonexpansive mappings by the authors in [5]. The proof of this important theorem is of the existential nature and does not describe any algorithm for constructing a common fixed point of an asymptotic pointwise $\rho$-nonexpansive semigroup. The current paper aims at filling this gap. The results of this paper generalize the conver-

(C2015 Bin Dehaish et al.; licensee Springer. This is an Open Access article distributed under the terms of the Creative Commons Attribution License (http://creativecommons.org/licenses/by/2.0), which permits unrestricted use, distribution, and reproduction in any medium, provided the original work is properly cited. 
gence of generalized Mann processes to common fixed points of semigroups of nonexpansive semigroups studied in the recent paper by Bin Dehaish and Kozlowski [6].

Let us recall that modular function spaces are a natural generalization of both function and sequence variants of many spaces like Lebesgue, Orlicz, Musielak-Orlicz, Lorentz, Orlicz-Lorentz, Calderon-Lozanovskii spaces and many others, important from an applications perspective; see the book by Kozlowski [7] for an extensive list of examples and special cases. There exists an extensive literature on the topic of the fixed point theory in modular function spaces; see e.g. [3, 7-18] and the references therein. It is also worthwhile mentioning a growing interest in applications of the methods of the fixed point theory to semigroups of nonlinear mappings and applications to the area of differential and integral equations (see e.g. $[10,19,20]$ ).

It is well known that the fixed point construction iteration processes for generalized nonexpansive mappings have been successfully used to develop efficient and powerful numerical methods for solving various nonlinear equations and variational problems, often of great importance for applications in various areas of pure and applied science. There exists an extensive literature on the subject of iterative fixed point construction processes for asymptotically nonexpansive mappings in Hilbert, Banach, and metric spaces; see e.g. [2, 21-37] and the references therein. Kozlowski proved convergence to a fixed point of some iterative algorithms of asymptotic pointwise nonexpansive mappings in Banach spaces [38] and the existence of common fixed points of semigroups of pointwise Lipschitzian mappings in Banach spaces [39]. Recently, the weak and strong convergence of such processes to common fixed points of semigroups of mappings in Banach spaces was demonstrated by Kozlowski and Sims [40] and by Kozlowski in [41].

We would like to emphasize that all convergence theorems proved in this paper define constructive algorithms that can be actually implemented. When dealing with specific applications of these theorems, one should take into consideration how additional properties of the mappings, sets, and modulars involved can influence the actual implementation of the algorithms defined in this paper.

\section{Preliminaries}

Let us introduce basic notions related to modular function spaces and related notation which will be used in this paper. For further details we refer the reader to preliminary sections of the recent articles [3, 6, 16] or to the survey article [17]; see also [7, 42, 43] for the standard framework of modular function spaces.

Let $\Omega$ be a nonempty set and $\Sigma$ be a nontrivial $\sigma$-algebra of subsets of $\Omega$. Let $\mathcal{P}$ be a $\delta$-ring of subsets of $\Omega$, such that $E \cap A \in \mathcal{P}$ for any $E \in \mathcal{P}$ and $A \in \Sigma$. Let us assume that there exists an increasing sequence of sets $K_{n} \in \mathcal{P}$ such that $\Omega=\bigcup K_{n}$. By $\mathcal{E}$ we denote the linear space of all simple functions with supports from $\mathcal{P}$. By $\mathcal{M}_{\infty}$ we will denote the space of all extended measurable functions, i.e. all functions $f: \Omega \rightarrow[-\infty, \infty]$ such that there exists a sequence $\left\{g_{n}\right\} \subset \mathcal{E},\left|g_{n}\right| \leq|f|$ and $g_{n}(\omega) \rightarrow f(\omega)$ for all $\omega \in \Omega$. By $1_{A}$ we denote the characteristic function of the set $A$.

Definition 2.1 [7] Let $\rho: \mathcal{M}_{\infty} \rightarrow[0, \infty]$ be a nontrivial, convex and even function. We say that $\rho$ is a regular convex function pseudomodular if

(i) $\rho(0)=0$;

(ii) $\rho$ is monotone, i.e. $|f(\omega)| \leq|g(\omega)|$ for all $\omega \in \Omega$ implies $\rho(f) \leq \rho(g)$, where $f, g \in \mathcal{M}_{\infty}$ 
(iii) $\rho$ is orthogonally subadditive, i.e. $\rho\left(f 1_{A \cup B}\right) \leq \rho\left(f 1_{A}\right)+\rho\left(f 1_{B}\right)$ for any $A, B \in \Sigma$ such that $A \cap B \neq \emptyset, f \in \mathcal{M}$;

(iv) $\rho$ has the Fatou property, i.e. $\left|f_{n}(\omega)\right| \uparrow|f(\omega)|$ for all $\omega \in \Omega$ implies $\rho\left(f_{n}\right) \uparrow \rho(f)$, where $f \in \mathcal{M}_{\infty}$;

(v) $\rho$ is order continuous in $\mathcal{E}$, i.e. $g_{n} \in \mathcal{E}$ and $\left|g_{n}(\omega)\right| \downarrow 0$ implies $\rho\left(g_{n}\right) \downarrow 0$.

Similarly to the case of measure spaces, we say that a set $A \in \Sigma$ is $\rho$-null if $\rho\left(g 1_{A}\right)=0$ for every $g \in \mathcal{E}$. We say that a property holds $\rho$-almost everywhere if the exceptional set is $\rho$-null. As usual we identify any pair of measurable sets whose symmetric difference is $\rho$-null as well as any pair of measurable functions differing only on a $\rho$-null set. With this in mind we define $\mathcal{M}=\left\{f \in \mathcal{M}_{\infty} ;|f(\omega)|<\infty \rho\right.$-a.e. $\}$, where each element is actually an equivalence class of functions equal $\rho$-a.e. rather than an individual function.

Definition 2.2 [7] We say that a regular function pseudomodular $\rho$ is a regular convex function modular if $\rho(f)=0$ implies $f=0 \rho$-a.e. The class of all nonzero regular convex function modulars defined on $\Omega$ will be denoted by $\Re$.

Definition 2.3 $[7,42,43]$ Let $\rho$ be a convex function modular. A modular function space is the vector space $L_{\rho}=\{f \in \mathcal{M} ; \rho(\lambda f) \rightarrow 0$ as $\lambda \rightarrow 0\}$. In the vector space $L_{\rho}$, the following formula:

$$
\|f\|_{\rho}=\inf \left\{\alpha>0 ; \rho\left(\frac{f}{\alpha}\right) \leq 1\right\}
$$

defines a norm, frequently called the Luxemburg norm.

The following notions will be used throughout the paper.

Definition 2.4 [8] Let $\rho \in \Re$.

(a) We say that $\left\{f_{n}\right\}$ is $\rho$-convergent to $f$ and write $f_{n} \rightarrow f(\rho)$ if and only if $\rho\left(f_{n}-f\right) \rightarrow 0$.

(b) A sequence $\left\{f_{n}\right\}$, where $f_{n} \in L_{\rho}$, is called $\rho$-Cauchy if $\rho\left(f_{n}-f_{m}\right) \rightarrow 0$ as $n, m \rightarrow \infty$.

(c) We say that $L_{\rho}$ is $\rho$-complete if and only if any $\rho$-Cauchy sequence in $L_{\rho}$ is $\rho$-convergent.

(d) A set $B \subset L_{\rho}$ is called $\rho$-closed if for any sequence of $f_{n} \in B$, the convergence $f_{n} \rightarrow f(\rho)$ implies that $f$ belongs to $B$.

(e) A set $B \subset L_{\rho}$ is called $\rho$-bounded if $\sup \{\rho(f-g) ; f \in B, g \in B\}<\infty$.

Since $\rho$ fails in general the triangle identity, many of the known properties of limit may not extend to $\rho$-convergence. For example, $\rho$-convergence does not necessarily imply the $\rho$-Cauchy condition. However, it is important to remember that the $\rho$-limit is unique when it exists. The following proposition brings together a few facts, which will be often used in the proofs of our results.

Proposition 2.1 [7] Let $\rho \in \Re$.

(i) $L_{\rho}$ is $\rho$-complete.

(ii) $\rho$-Balls $B_{\rho}(f, r)=\left\{g \in L_{\rho} ; \rho(f-g) \leq r\right\}$ are $\rho$-closed.

(iii) If $\rho\left(\alpha f_{n}\right) \rightarrow 0$ for an $\alpha>0$ then there exists a subsequence $\left\{g_{n}\right\}$ of $\left\{f_{n}\right\}$ such that $g_{n} \rightarrow 0 \rho$-a.e. 
(iv) $\rho(f) \leq \liminf _{n \rightarrow \infty} \rho\left(f_{n}\right)$ whenever $f_{n} \rightarrow f \rho$-a.e. (note: this property is equivalent to the Fatou property).

Let us recall the definition of an asymptotic pointwise nonexpansive mapping acting in a modular function space.

Definition 2.5 [3] Let $\rho \in \Re$ and let $C \subset L_{\rho}$ be nonempty and $\rho$-closed. A mapping $T$ : $C \rightarrow C$ is called

(i) a pointwise Lipschitzian mapping if there exists $\alpha: C \rightarrow[0, \infty)$ such that

$$
\rho(T(f)-T(g)) \leq \alpha(f) \rho(f-g) \quad \text { for any } f, g \in C \text {; }
$$

(ii) an asymptotic pointwise nonexpansive if there exists a sequence of mappings $\alpha_{n}: C \rightarrow[0, \infty)$ such that

$$
\rho\left(T^{n}(f)-T^{n}(g)\right) \leq \alpha_{n}(f) \rho(f-g) \quad \text { for any } f, g \in C \text {. }
$$

and $\limsup _{n \rightarrow \infty} \alpha_{n}(f) \leq 1$ for any $f \in L_{\rho}$.

A point $f \in C$ is called a fixed point of $T$ whenever $T(f)=f$. The set of fixed points of $T$ will be denoted by $F(T)$.

Define $b_{n}(f)=a_{n}(f)-1$, where $a_{n}(f)=\max \left\{\alpha_{n}(f), 1\right\}$, for any $f \in C$ and $n \in \mathbb{N}$. Clearly then

$$
\lim _{n \rightarrow \infty} b_{n}(f)=0
$$

Definition 2.6 Define $\mathcal{T}_{r}(C)$ as a class of all asymptotic pointwise nonexpansive mappings $T$ such that

$$
\sum_{n=1}^{\infty} b_{n}(f)<\infty \quad \text { for every } f \in C,
$$

$a_{n}$ is a bounded function for every $n \geq 1$.

The notion of the asymptotic pointwise nonexpansiveness will be now extended to a one-parameter family of mappings. Throughout this paper $J$ will be the semigroup of all nonnegative numbers, that is, $J=[0, \infty)$ with normal addition.

Definition 2.7 A one-parameter family $\mathcal{F}=\left\{T_{t}: t \in J\right\}$ of mappings from $C$ into itself is said to be an asymptotic pointwise nonexpansive semigroup on $C$ if $\mathcal{F}$ satisfies the following conditions:

(i) $T_{0}(f)=f$ for $f \in C$;

(ii) $T_{t+s}(f)=T_{t}\left(T_{s}(f)\right)$ for $f \in C$ and $t, s \in[0, \infty)$;

(iii) for each $t \geq 0, T_{t}$ is an asymptotic pointwise nonexpansive mapping, i.e. there exists a function $\alpha_{t}: C \rightarrow[0, \infty)$ such that

$$
\rho\left(T_{t}(f)-T_{t}(g)\right) \leq \alpha_{t}(f) \rho(f-g) \quad \text { for all } f, g \in C \text {, }
$$

such that $\lim \sup _{t \rightarrow \infty} \alpha_{t}(f) \leq 1$ for every $f \in C$; 
(iv) for each $f \in C$, the mapping $t \rightarrow T_{t}(f)$ is $\rho$-continuous.

For each $t \in J$ let $F\left(T_{t}\right)$ denote the set of its fixed points. Define then the set of all common fixed points set for mappings from $\mathcal{F}$ as the following intersection:

$$
F(\mathcal{F})=\bigcap_{t \in J} F\left(T_{t}\right) .
$$

The common fixed points are frequently interpreted as the stationary points of the semigroup $\mathcal{F}$. Note that without loss of generality we may assume $\alpha_{t}(f) \geq 1$ for any $t \in J$ and $f \in C$ and $\limsup _{t \rightarrow \infty} \alpha_{t}(f)=\lim _{t \rightarrow \infty} \alpha_{t}(f)=1$.

Denoting $a_{0} \equiv 1$ and $a_{t}(f)=\max \left(\alpha_{t}(f), 1\right)$ for $t>0$, we note that without loss of generality we can assume that $\mathcal{F}$ is asymptotically nonexpansive if

$$
\begin{aligned}
& \rho\left(T_{t}(f)-T_{t}(g)\right) \leq a_{t}(f) \rho(f-g) \quad \text { for all } f, g \in C, t \in J, \\
& \lim _{t \rightarrow \infty} a_{t}(f)=1, \quad a_{t}(f) \geq 1 \quad \text { for all } f \in C \text { and } t \in J .
\end{aligned}
$$

Define $b_{t}(f)=a_{t}(f)-1$. Note that

$$
\lim _{t \rightarrow \infty} b_{t}(f)=0
$$

The above notation will be consistently used throughout this paper.

Definition 2.8 By $\mathcal{S}(C)$ we will denote the class of all asymptotic pointwise nonexpansive semigroups on $C$ such that

$$
\begin{aligned}
& M_{t}=\sup \left\{a_{t}(f): f \in C\right\}<\infty \quad \text { for every } t \in J, \\
& \limsup _{t \rightarrow \infty} M_{t}=1 .
\end{aligned}
$$

Note that we do not assume that all functions $a_{t}$ are bounded by a common constant. Therefore, we do not assume that $\mathcal{F}$ is uniformly Lipschitzian.

Definition 2.9 We will say that a semigroup $\mathcal{F} \in \mathcal{S}(C)$ is $\rho$ continuous if

$$
\lim _{t \rightarrow t_{0}} \rho\left(T_{t}(f)-T_{t_{0}}(f)\right)=0
$$

for any $f \in C$ and $t_{0} \in[0,+\infty)$.

The concept $\rho$-type is a powerful technical tool which is used in the proofs of many fixed point results. The definition of a $\rho$-type is based on a given sequence. In this work, we generalize this definition to be adapted to one-parameter family of mappings.

Definition 2.10 Let $K \subset L_{\rho}$ be convex and $\rho$-bounded.

(1) A function $\tau: K \rightarrow[0, \infty]$ is called a $\rho$-type (or shortly a type) if there exists a one-parameter family $\left\{h_{t}\right\}_{t \geq 0}$ of elements of $K$ such that for any $f \in K$ we have

$$
\tau(f)=\inf _{M>0}\left(\sup _{t \geq M} \rho\left(h_{t}-f\right)\right) .
$$


(2) Let $\tau$ be a $\rho$-type. A sequence $\left\{g_{n}\right\}$ is called a minimizing sequence of $\tau$ if

$$
\lim _{n \rightarrow \infty} \tau\left(g_{n}\right)=\inf \{\tau(f): f \in K\}
$$

Note that $\tau$ is convex provided $\rho$ is convex.

Let us recall the modular equivalents of uniform convexity introduced in [3].

Definition 2.11 Let $\rho \in \Re$. We define the following uniform convexity (UC) type properties of the function modular $\rho$ :

(i) Let $r>0, \varepsilon>0$. Define

$$
D(r, \varepsilon)=\left\{(f, g): f, g \in L_{\rho}, \rho(f) \leq r, \rho(g) \leq r, \rho(f-g) \geq \varepsilon r\right\} .
$$

Let

$$
\delta(r, \varepsilon)=\inf \left\{1-\frac{1}{r} \rho\left(\frac{f+g}{2}\right):(f, g) \in D(r, \varepsilon)\right\}, \quad \text { if } D(r, \varepsilon) \neq \emptyset \text {, }
$$

and $\delta(r, \varepsilon)=1$ if $D(r, \varepsilon)=\emptyset$. We say that $\rho$ satisfies (UC) if for every $r>0, \varepsilon>0$, $\delta(r, \varepsilon)>0$. Note that for every $r>0, D(r, \varepsilon) \neq \emptyset$, for $\varepsilon>0$ small enough.

(ii) We say that $\rho$ satisfies (UUC1) if there exists $\eta(s, \varepsilon)>0$, for every $s \geq 0$, and $\varepsilon>0$ such that

$$
\delta(r, \varepsilon)>\eta(s, \varepsilon)>0 \quad \text { for } r>s .
$$

We will need the following result, being a modular equivalent of a norm property in uniformly convex Banach spaces; see e.g. [26].

Lemma 2.1 [6] Let $\rho \in \Re$ be (UUC1) and let $\left\{c_{k}\right\} \subset(0,1)$ be bounded away from 0 and 1 . If there exists $R>0$ such that

$$
\begin{aligned}
& \limsup _{n \rightarrow \infty} \rho\left(f_{n}\right) \leq R, \quad \limsup _{n \rightarrow \infty} \rho\left(g_{n}\right) \leq R, \\
& \lim _{n \rightarrow \infty} \rho\left(c_{n} f_{n}+\left(1-c_{n}\right) g_{n}\right)=R,
\end{aligned}
$$

then

$$
\lim _{n \rightarrow \infty} \rho\left(f_{n}-g_{n}\right)=0
$$

The following property plays in the theory of modular function space a role similar to the reflexivity in Banach spaces; see e.g. [9].

Definition 2.12 We say that $L_{\rho}$ has property $(R)$ if and only if every nonincreasing sequence $\left\{C_{n}\right\}$ of nonempty, $\rho$-bounded, $\rho$-closed, and convex subsets of $L_{\rho}$ has nonempty intersection.

Similarly to the Banach space case, the modular uniform convexity implies property $(R)$. 
Theorem 2.1 [3] Let $\rho \in \Re$ be (UUC1) then $L_{\rho}$ has property $(R)$.

The next lemma is a generalization of the minimizing sequence property for types defined by sequences in Lemma 4.3 in [16] to the one-parameter semigroup case.

Lemma 2.2 [5] Assume $\rho \in \Re$ is (UUC1). Let $C$ be a nonempty, $\rho$-bounded, $\rho$-closed, and convex subset of $L_{\rho}$. Let $\tau$ be a type defined by a one-parameter family $\left\{h_{t}\right\}_{t \geq 0}$ in $C$.

(i) If $\tau\left(f_{1}\right)=\tau\left(f_{2}\right)=\inf _{f \in C} \tau(f)$, then $f_{1}=f_{2}$.

(ii) Any minimizing sequence $\left\{f_{n}\right\}$ of $\tau$ is $\rho$-convergent. Moreover, the $\rho$-limit of $\left\{f_{n}\right\}$ is independent of the minimizing sequence.

Using Lemma 2.2, the authors proved the following common fixed point result for asymptotic pointwise nonexpansive semigroups.

Theorem 2.2 [5] Assume $\rho \in \Re$ is (UUC1). Let $C$ be a $\rho$-closed, $\rho$-bounded, convex, and nonempty subset. Let $\mathcal{F}=\left\{T_{t}: t \in J\right\}$ be an asymptotic pointwise nonexpansive semigroup on $C$. Then $\mathcal{F}$ has a common fixed point and the set $F(\mathcal{F})$ of common fixed points is $\rho$-closed and convex.

\section{The demiclosedness principle}

In this section we will use the notion the uniform continuity of the function modular $\rho$ in the sense of the following definition (see e.g. [16]).

Definition 3.1 We say that $\rho \in \Re$ is uniformly continuous if for every $\varepsilon>0$ and $L>0$, there exists $\delta>0$ such that

$$
|\rho(g)-\rho(g+h)| \leq \varepsilon
$$

provided $\rho(h)<\delta$ and $\rho(g) \leq L$.

Let us mention that the uniform continuity holds for a large class of function modulars. For instance, it can be proved that in Orlicz spaces over a finite atomless measure [44] or in Orlicz sequence spaces [45] the uniform continuity of the Orlicz modular is equivalent to the $\Delta_{2}$-type condition. Recall that $\rho$ satisfies the $\Delta_{2}$-type condition if and only if there exists $K>0$ such that $\rho(2 f) \leq K \rho(f)$, for any $f \in L_{\rho}$.

Let us recall the definition of the Opial property and the strong Opial property in modular function spaces $[16,46]$.

Definition 3.2 We say that $L_{\rho}$ satisfies the $\rho$-a.e. Opial property if for every $\left\{f_{n}\right\} \in L_{\rho}$ which is $\rho$-a.e. convergent to 0 such that there exists a $\beta>1$ for which

$$
\sup _{n}\left\{\rho\left(\beta f_{n}\right)\right\}<\infty
$$

the following inequality holds for any $g \in E_{\rho}$ not equal to 0 :

$$
\liminf _{n \rightarrow \infty} \rho\left(f_{n}\right) \leq \liminf _{n \rightarrow \infty} \rho\left(f_{n}+g\right) .
$$


Definition 3.3 We say that $L_{\rho}$ satisfies the $\rho$-a.e. strong Opial property if for every $\left\{f_{n}\right\} \in$ $L_{\rho}$ which is $\rho$-a.e. convergent to 0 such that there exists a $\beta>1$ for which

$$
\sup _{n}\left\{\rho\left(\beta f_{n}\right)\right\}<\infty
$$

the following equality holds for any $g \in E_{\rho}$ :

$$
\liminf _{n \rightarrow \infty} \rho\left(f_{n}+g\right)=\liminf _{n \rightarrow \infty} \rho\left(f_{n}\right)+\rho(g)
$$

Remark 3.1 Note that the $\rho$-a.e. strong Opial property implies the $\rho$-a.e. Opial property [46].

Remark 3.2 Also, note that, by virtue of Theorem 2.1 in [46], every convex, orthogonally additive function modular $\rho$ has the $\rho$-a.e. strong Opial property. Let us recall that $\rho$ is called orthogonally additive if $\rho(f, A \cup B)=\rho(f, A)+\rho(f, B)$ whenever $A \cap B=\emptyset$. Therefore, all Orlicz and Musielak-Orlicz spaces must have the strong Opial property.

Note that the Opial property in the norm sense does not necessarily hold for several classical Banach function spaces. For instance the norm Opial property does not hold for $L^{p}$ spaces for $1 \leq p \neq 2$, while the modular strong Opial property holds in $L^{p}$ for all $p \geq 1$.

Lemma 3.1 [4] Let $\rho \in \Re$. Assume that $L_{\rho}$ has the $\rho$-a.e. strong Opial property. Let $C \subset$ $E_{\rho}$ be a nonempty, strongly $\rho$-bounded, and $\rho$-a.e. compact convex set. Then any $\rho$-type defined in $C$ attains its minimum in $C$.

To begin our discussion of the demiclosedness principle, let us quote the following version of this theorem applied to the asymptotic pointwise nonexpansive mappings [6, Theorem 4.1].

Theorem 3.1 (Demiclosedness principle) Let $\rho \in \Re$. Assume that

(1) $\rho$ is (UUC1),

(2) $\rho$ has the strong Opial property,

(3) $\rho$ has the $\Delta_{2}$ property and is uniformly continuous.

Let $C \subset L_{\rho}$ be nonempty, convex, strongly $\rho$-bounded, and $\rho$-closed, and let $T \in \mathcal{T}_{r}(C)$. Let $\left\{x_{n}\right\} \subset C$, and $x \in C$. If $x_{n} \rightarrow x \rho$-a.e. and $\rho\left(T\left(x_{n}\right)-x_{n}\right) \rightarrow 0$, then $x \in F(T)$.

We will need a version of the above theorem without assuming that $T \in \mathcal{T}_{r}(C)$. This will require a different proof, which is sketched below.

Theorem 3.2 Let $\rho \in \Re$. Assume that

(1) $\rho$ is (UUC1),

(2) $\rho$ has the strong Opial property,

(3) $\rho$ has the $\Delta_{2}$ property and is uniformly continuous.

Let $C \subset L_{\rho}$ be nonempty, convex, strongly $\rho$-bounded, and $\rho$-closed. Let $T: C \rightarrow C$ be an asymptotic pointwise nonexpansive mapping such that

$$
\rho\left(T^{n}(f)-T^{n}(g)\right) \leq \alpha_{n}(f) \rho(f-g) \quad \text { for any } f, g \in C,
$$


and $\lim \sup _{n \rightarrow \infty} \alpha_{n}(f) \leq 1$ for any $f \in L_{\rho}$. We will assume the functions $\left\{\alpha_{n}\right\}$ are bounded on $C$, i.e. $T$ is uniformly $\rho$-Lipschitzian mapping. Let $\left\{f_{n}\right\} \subset C$, and $f \in C$. If $f_{n} \rightarrow f \rho$-a.e. and $\rho\left(T\left(f_{n}\right)-f_{n}\right) \rightarrow 0$, then $f \in F(T)$. In particular, we have $f \in \bigcap_{n \geq 1} F\left(T^{n}\right)$.

Proof Define the $\rho$-type function

$$
\tau(g)=\limsup _{n \rightarrow \infty} \rho\left(f_{n}-g\right), \quad g \in C .
$$

Note that

$$
\lim _{n \rightarrow \infty} \rho\left(T^{m}\left(f_{n}\right)-f_{n}\right)=0, \quad m=1,2, \ldots
$$

Indeed, we have

$$
\rho\left(\frac{T^{m}\left(f_{n}\right)-f_{n}}{m}\right) \leq \frac{1}{m} \sum_{k=0}^{k=m-1} \rho\left(T^{k+1}\left(f_{n}\right)-T^{k}\left(f_{n}\right)\right),
$$

which implies

$$
\begin{aligned}
\rho\left(\frac{T^{m}\left(f_{n}\right)-f_{n}}{m}\right) & \leq \frac{1}{m} \sum_{k=0}^{k=m-1} \alpha_{k}\left(f_{n}\right) \rho\left(T\left(f_{n}\right)-f_{n}\right) \\
& \leq\left(\sup _{g \in C, k \in[0, m-1]} \alpha_{k}(g)\right) \rho\left(T\left(f_{n}\right)-f_{n}\right)
\end{aligned}
$$

for any $n \geq 1$, where $\alpha_{0}=1$. Hence

$$
\lim _{n \rightarrow \infty} \rho\left(\frac{T^{m}\left(f_{n}\right)-f_{n}}{m}\right)=0, \quad m=1,2, \ldots
$$

Since $\rho$ has the $\Delta_{2}$ property, we get

$$
\lim _{n \rightarrow \infty} \rho\left(T^{m}\left(f_{n}\right)-f_{n}\right)=0, \quad m=1,2, \ldots
$$

Since $\rho$ is uniformly continuous, we get

$$
\tau(g)=\limsup _{n \rightarrow \infty} \rho\left(f_{n}-g\right)=\limsup _{n \rightarrow \infty} \rho\left(T^{m}\left(f_{n}\right)-g\right)
$$

for any $g \in C$ and $m \geq 1$. In particular, we have

$$
\limsup _{n \rightarrow \infty} \rho\left(T^{m}\left(f_{n}\right)-T^{m}(f)\right) \leq \alpha_{m}(f) \limsup _{n \rightarrow \infty} \rho\left(f_{n}-f\right), \quad m=1,2, \ldots
$$

In other words, we have $\tau\left(T^{m}(f)\right) \leq \alpha_{m}(f) \tau(f)$, for any $m \geq 1$. Since $\rho$ has the Opial property, it is easy to prove that

$$
\tau(f)=\inf \{\tau(g): g \in C\} .
$$


Since $T$ is an asymptotic pointwise nonexpansive mapping, we get

$$
\lim _{m \rightarrow \infty} \tau\left(T^{m}(f)\right)=\tau(f)
$$

Since $\rho$ is (UUC1), then arguing similarly to the proof of Theorem 4.1 in [6], we have

$$
\lim _{m \rightarrow \infty} \rho\left(T^{m}(f)-f\right)=0
$$

Since $T$ is $\rho$-continuous, we get $T(f)=f$, i.e. $f \in F(T)$.

As a corollary to this result, we get the following important result.

\section{Corollary 3.1 Let $\rho \in \Re$. Assume that}

(1) $\rho$ is (UUC1),

(2) $\rho$ has the strong Opial property,

(3) $\rho$ has the $\Delta_{2}$ property and is uniformly continuous.

Let $C \subset L_{\rho}$ be nonempty, convex, strongly $\rho$-bounded, and $\rho$-closed. Let $T, S: C \rightarrow C$ be asymptotic pointwise nonexpansive mappings such that

$$
\rho\left(T^{n}(f)-T^{n}(g)\right) \leq \alpha_{n}(f) \rho(f-g) \quad \text { and } \quad \rho\left(S^{n}(f)-S^{n}(g)\right) \leq \beta_{n}(f) \rho(f-g)
$$

for any $f, g \in C$, with $\limsup _{n \rightarrow \infty} \alpha_{n}(f) \leq 1$, and $\limsup _{n \rightarrow \infty} \beta_{n}(f) \leq 1$, for any $f \in L_{\rho}$. We will assume the functions $\left\{\alpha_{n}\right\}$ and $\left\{\beta_{n}\right\}$ are bounded on C. Let $\left\{f_{n}\right\} \subset C$, and $f \in C$.Iff $\rightarrow f$ $\rho$-a.e. and

$$
\lim _{n \rightarrow \infty} \rho\left(T\left(f_{n}\right)-f_{n}\right)=\lim _{n \rightarrow \infty} \rho\left(S\left(f_{n}\right)-f_{n}\right)=0,
$$

then $f \in F(T) \cap F(S)$. In particular, we have $f \in \bigcap_{n, m \geq 1} F\left(T^{n} \circ S^{m}\right)$.

The above results lead us to the following version of the demiclosedness principle for semigroup of mappings.

Theorem 3.3 (Demiclosedness principle) Let $\rho \in \Re$. Assume that

(1) $\rho$ is (UUC1),

(2) $\rho$ has the strong Opial property,

(3) $\rho$ has the $\Delta_{2}$ property and is uniformly continuous.

Let $C \subset L_{\rho}$ be nonempty, convex, strongly $\rho$-bounded, and $\rho$-closed, and let $\mathcal{F} \in \mathcal{S}(C)$ be continuous. Let $\left\{f_{n}\right\} \subset C$, and $f \in C$. Assume $f_{n} \rightarrow f \rho$-a.e. If there exist $t_{1}, t_{2} \in[0,+\infty)$ such that $\frac{t_{1}}{t_{2}}$ is irrational and

$$
\lim _{n \rightarrow \infty} \rho\left(T_{t_{1}}\left(f_{n}\right)-f_{n}\right)=\lim _{n \rightarrow \infty} \rho\left(T_{t_{2}}\left(f_{n}\right)-f_{n}\right)=0
$$

then $f \in F(\mathcal{F})$.

Proof In view of Corollary 3.1, we know that

$$
f \in F\left(T_{t_{1}}\right) \cap F\left(T_{t_{2}}\right) .
$$


Let us denote $G_{+}\left(t_{1}, t_{2}\right)=\left\{n t_{1}+m t_{2} \geq 0: n, m \in \mathbb{N} \cup\{0\}\right\}$. Note that for any $n, m \in \mathbb{N} \cup\{0\}$ we have

$$
F\left(T_{t_{1}}\right) \cap F\left(T_{t_{2}}\right) \subset F\left(T_{n t_{1}+m t_{2}}\right) .
$$

Combining the above we get

$$
f \in F\left(T_{t_{1}}\right) \cap F\left(T_{t_{2}}\right) \subset \bigcap_{t \in G_{+}\left(t_{1}, t_{2}\right)} F\left(T_{t}\right) .
$$

Since $\frac{t_{1}}{t_{2}}$ is irrational, then the set $G_{+}\left(t_{1}, t_{2}\right)$ is dense in $[0,+\infty)$ [47]. Since $\mathcal{F}$ is continuous and $\rho$ is uniformly continuous, we have

$$
F(\mathcal{F})=\bigcap_{t \in G_{+}\left(t_{1}, t_{2}\right)} F\left(T_{t}\right)
$$

Hence $f \in F(\mathcal{F})$ as desired.

\section{Convergence of generalized Krasnosel'skii-Mann iteration processes}

Let us start with the precise definition of the generalized Krasnosel'skii-Mann iteration process for semigroups of nonlinear mappings.

Definition 4.1 Let $\mathcal{F} \in \mathcal{S}(\mathcal{C}),\left\{t_{k}\right\} \subset J$ and $\left\{c_{k}\right\} \subset(0,1)$. The generalized Krasnosel'skiiMann iteration process $\operatorname{gKM}\left(\mathcal{F},\left\{c_{k}\right\},\left\{t_{k}\right\}\right)$ generated by the semigroup $\mathcal{F}$, the sequences $\left\{c_{k}\right\}$ and $\left\{t_{k}\right\}$, is defined by the following iterative formula:

$$
x_{k+1}=c_{k} T_{t_{k}}\left(x_{k}\right)+\left(1-c_{k}\right) x_{k}, \quad \text { where } x_{1} \in C \text { is chosen arbitrarily }
$$

and

(1) $\left\{c_{k}\right\}$ is bounded away from 0 and 1 ,

(2) $\lim _{k \rightarrow \infty} t_{k}=\infty$,

(3) $\sum_{n=1}^{\infty} b_{t_{n}}(x)<\infty$ for every $x \in C$.

Definition 4.2 We say that a generalized Krasnosel'skii-Mann iteration process $\operatorname{gKM}(\mathcal{F}$, $\left.\left\{c_{k}\right\},\left\{t_{k}\right\}\right)$ is well defined if

$$
\limsup _{k \rightarrow \infty} a_{t_{k}}\left(x_{k}\right)=1 \text {. }
$$

Arguing exactly like in the proof of Lemma 5.2 in [6] (see also Lemma 22.20 in [40]), we get the following result.

Lemma 4.1 Let $\rho \in \Re$ be (UUC1). Let $C \subset L_{\rho}$ be a $\rho$-closed, $\rho$-bounded, and convex set. Let $\mathcal{F} \in \mathcal{S}(C), w \in F(\mathcal{F})$, and let $\left\{x_{k}\right\}$ be a sequence generated by a generalized Krasnosel'skiiMann process $\operatorname{gKM}\left(\mathcal{F},\left\{c_{k}\right\},\left\{t_{k}\right\}\right)$. Then there exists an $r \in \mathbb{R}$ such that $\lim _{k \rightarrow \infty} \rho\left(x_{k}-w\right)=r$.

We will prove now a generic version of the convergence theorem for the sequences $\left\{x_{k}\right\}$ which are generated by the Krasnosel'skii-Mann iteration process and are at the same time approximate fixed point sequences. 
Theorem 4.1 Let $\rho \in \Re$. Assume that

(1) $\rho$ is (UUC1),

(2) $\rho$ has the strong Opial property,

(3) $\rho$ has the $\Delta_{2}$ property and is uniformly continuous.

Let $C \subset L_{\rho}$ be nonempty, $\rho$-a.e.compact, convex, strongly $\rho$-bounded, and $\rho$-closed, and let $\mathcal{F} \in \mathcal{S}(C)$. Assume that $\operatorname{gKM}\left(\mathcal{F},\left\{c_{k}\right\},\left\{t_{k}\right\}\right)$ is a well defined Krasnosel'skii-Mann iteration process. If for the sequence $\left\{x_{k}\right\}$ generated by $\operatorname{gKM}\left(\mathcal{F},\left\{c_{k}\right\},\left\{t_{k}\right\}\right)$ we have

$$
\rho\left(T_{s_{1}}\left(x_{k}\right)-x_{k}\right) \rightarrow 0, \quad \rho\left(T_{s_{2}}\left(x_{k}\right)-x_{k}\right) \rightarrow 0,
$$

where $s_{1}, s_{2} \in[0,+\infty)$ are such that $\frac{s_{1}}{s_{2}}$ is irrational, then $\left\{x_{k}\right\}$ converges $\rho$-a.e. to a common fixed point $w \in F(\mathcal{F})$.

Proof Observe that by Theorem 2.2 the set of fixed points $F(\mathcal{F})$ is nonempty, convex and $\rho$-closed. Consider $y, z \in C$, two $\rho$-a.e. cluster points of $\left\{x_{k}\right\}$. There exits then $\left\{y_{k}\right\},\left\{z_{k}\right\}$ subsequences of $\left\{x_{k}\right\}$ such that $y_{k} \rightarrow y \rho$-a.e., and $z_{k} \rightarrow z \rho$-a.e. It follows from Theorem 3.3 that $T_{s_{1}}(y)=y$ and $T_{S_{1}}(z)=z$. By Lemma 4.1, there exist $r_{y}, r_{z} \in \mathbb{R}$ such that

$$
r_{y}=\lim _{k \rightarrow+\infty} \rho\left(x_{k}-y\right), \quad r_{z}=\lim _{k \rightarrow+\infty} \rho\left(x_{k}-z\right)
$$

We claim that $y=z$. Assume to the contrary that $y \neq z$. Then by the Opial property we have

$$
\begin{aligned}
r_{y} & =\liminf _{k \rightarrow \infty} \rho\left(y_{k}-y\right)<\liminf _{k \rightarrow \infty} \rho\left(y_{k}-z\right) \\
& =\liminf _{k \rightarrow \infty} \rho\left(z_{k}-z\right)<\liminf _{k \rightarrow \infty} \rho\left(z_{k}-y\right)=r_{y} .
\end{aligned}
$$

The contradiction implies that $y=z$. Therefore, $\left\{x_{k}\right\}$ has at most one $\rho$-a.e. cluster point. Since $C$ is $\rho$-a.e. compact it follows that the sequence $\left\{x_{k}\right\}$ has exactly one $\rho$-a.e. cluster point $w \in C$, which means that $x_{k} \rightarrow w \rho$-a.e. Applying the demiclosedness principle again, we get $T_{s_{1}}(w)=w$. By the same argument, we get $T_{s_{2}}(w)=w$ (observe that the construction of $w$ did not depend on the selection of $\left.s_{i}\right)$. From the density of $G_{+}\left(t_{1}, t_{2}\right)$ in $J=[0,+\infty)$, we conclude that $T_{t}(w)=w$ for any $t \in J$, as claimed.

Let us apply the above result to some more specific situations. First we need to prove a series of axillary results. Let us start with the following elementary lemma.

Lemma 4.2 [31] Suppose $\left\{r_{k}\right\}$ is a bounded sequence of real numbers and $\left\{d_{k, n}\right\}$ is a double index sequence of real numbers which satisfy

$$
\limsup _{k \rightarrow \infty} \limsup _{n \rightarrow \infty} d_{k, n} \leq 0 \text { and } \quad r_{k+n} \leq r_{k}+d_{k, n}
$$

for each $k, n \geq 1$. Then $\left\{r_{k}\right\}$ converges to an $r \in \mathbb{R}$.

The following result provides an important technique which will be used in this paper. 
Lemma 4.3 Let $\rho \in \Re$ be (UUC1). Let $C \subset L_{\rho}$ be a $\rho$-closed, $\rho$-bounded, and convex set. Let $\mathcal{F} \in \mathcal{S}(C)$. Assume that $w$ is a common fixed point of $\mathcal{F}$. Let us denote by $\left\{x_{k}\right\}$ a sequence generated by the generalized Krasnosel'skii-Mann process $\operatorname{gKM}\left(T,\left\{c_{k}\right\},\left\{t_{k}\right\}\right)$. Then there exists $r \in \mathbb{R}$ such that

$$
\lim _{k \rightarrow \infty} \rho\left(x_{k}-w\right)=r
$$

Proof Let $w \in F(\mathcal{F})$. Since

$$
\begin{aligned}
\rho\left(x_{k+1}-w\right) & \leq c_{k} \rho\left(T_{t_{k}}\left(x_{k}\right)-w\right)+\left(1-c_{k}\right) \rho\left(x_{k}-w\right) \\
& =c_{k} \rho\left(T_{t_{k}}\left(x_{k}\right)-T_{t_{k}}(w)\right)+\left(1-c_{k}\right) \rho\left(x_{k}-w\right) \\
& \leq c_{k}\left(1+b_{t_{k}}(w)\right) \rho\left(x_{k}-w\right)+\left(1-c_{k}\right) \rho\left(x_{k}-w\right) \\
& =c_{k} b_{t_{k}}(w) \rho\left(x_{k}-w\right)+\rho\left(x_{k}-w\right) \\
& \leq b_{t_{k}}(w) \operatorname{diam}_{\rho}(C)+\rho\left(x_{k}-w\right),
\end{aligned}
$$

it follows that for every $n \in \mathbb{N}$,

$$
\rho\left(x_{k+n}-w\right) \leq \rho\left(x_{k}-w\right)+\operatorname{diam}_{\rho}(C) \sum_{i=k}^{k+n-1} b_{t_{i}}(w) .
$$

Denote $r_{p}=\rho\left(x_{p}-w\right)$ for every $p \in \mathbb{N}$ and $d_{k, n}=\operatorname{diam}_{\rho}(C) \sum_{i=k}^{k+n-1} b_{t_{i}}(w)$. Observe that by the assumptions on the sequence $\left\{t_{n}\right\}, \lim \sup _{k \rightarrow \infty} \lim _{\sup _{n \rightarrow \infty}} d_{k, n}=0$. By Lemma 4.2, there exists an $r \in \mathbb{R}$ such that $\lim _{k \rightarrow \infty} \rho\left(x_{k}-w\right)=r$, as claimed.

Lemma 4.4 Let $\rho \in \Re$ be (UUC1). Let $C \subset L_{\rho}$ be a $\rho$-closed, $\rho$-bounded, and convex set, and $\mathcal{F} \in \mathcal{S}(C)$. Let $\operatorname{gKM}\left(T,\left\{c_{k}\right\},\left\{t_{k}\right\}\right)$ be a generalized Krasnosel'skii-Mann iteration process. Then

$$
\lim _{k \rightarrow \infty} \rho\left(T_{t_{k}}\left(x_{k}\right)-x_{k}\right)=0
$$

and

$$
\lim _{k \rightarrow \infty} \rho\left(x_{k+1}-x_{k}\right)=0
$$

Proof By Theorem 2.2, $\mathcal{F}$ has at least one common fixed point $w \in C$. In view of Lemma 4.3, there exists $r \in \mathbb{R}$ such that

$$
\lim _{k \rightarrow \infty} \rho\left(x_{k}-w\right)=r .
$$

Note that

$$
\begin{aligned}
\limsup _{k \rightarrow \infty} \rho\left(T_{t_{k}}\left(x_{k}\right)-w\right) & =\limsup _{k \rightarrow \infty} \rho\left(T_{t_{k}}\left(x_{k}\right)-T_{t_{k}}(w)\right) \\
& \leq \limsup _{k \rightarrow \infty} a_{t_{k}}(w) \rho\left(x_{k}-w\right) \leq r
\end{aligned}
$$


and that

$$
\lim _{k \rightarrow \infty} \rho\left(c_{k}\left(T_{t_{k}}\left(x_{k}\right)-w\right)+\left(1-c_{k}\right)\left(x_{k}-w\right)\right)=\lim _{k \rightarrow \infty} \rho\left(x_{k+1}-w\right)=r .
$$

Set $f_{k}=T_{t_{k}}\left(x_{k}\right)-w, g_{k}=x_{k}-w$, and note that $\limsup _{k \rightarrow \infty} \rho\left(g_{k}\right) \leq r$ by (4.10), and $\lim \sup _{k \rightarrow \infty} \rho\left(f_{k}\right) \leq r$ by (4.11). Observe also that

$$
\begin{aligned}
& \lim _{k \rightarrow \infty} \rho\left(c_{k} f_{k}+\left(1-c_{k}\right) g_{k}\right) \\
& \quad=\lim _{k \rightarrow \infty} \rho\left(c_{k} T_{t_{k}}\left(x_{k}\right)+\left(1-c_{k}\right) x_{k}-w\right) \\
& \quad=\lim _{k \rightarrow \infty} \rho\left(x_{k+1}-w\right)=r .
\end{aligned}
$$

Hence, it follows from Lemma 2.1 that

$$
\lim _{k \rightarrow \infty} \rho\left(T_{t_{k}}\left(x_{k}\right)-x_{k}\right)=\lim _{k \rightarrow \infty} \rho\left(f_{k}-g_{k}\right)=0,
$$

which by the construction of the sequence $\left\{x_{k}\right\}$ is equivalent to

$$
\lim _{k \rightarrow \infty} \rho\left(x_{k+1}-x_{k}\right)=0
$$

as claimed.

Lemma 4.5 Let $\rho \in \Re$ be (UUC1) and have the $\Delta_{2}$ property. Let $C \subset L_{\rho}$ be a $\rho$-closed, $\rho$-bounded, and convex set and let $\mathcal{F} \in \mathcal{S}(C)$. Denote by $\left\{x_{k}\right\}$ the sequence generated by a well defined generalized Krasnosel'skii-Mann process $\operatorname{gKM}\left(\mathcal{F},\left\{c_{k}\right\},\left\{t_{k}\right\}\right)$. Let $A \subset J$ be such that for every $s \in A$ there exists a strictly increasing sequence of natural numbers $\left\{j_{k}\right\}$ satisfying the following conditions:

(a) $\rho\left(x_{k}-x_{j_{k}}\right) \rightarrow 0$ as $k \rightarrow \infty$,

(b) $\lim _{k \rightarrow \infty} \rho\left(T_{d_{k}}\left(x_{j_{k}}\right)-x_{j_{k}}\right)=0$, where $d_{k}=\left|t_{j_{k+1}}-t_{j_{k}}-s\right|$.

Then $\left\{x_{k}\right\}$ is an approximate fixed point sequence for all mappings $\left\{T_{s}\right\}$ where $s \in A$, that is,

$$
\lim _{k \rightarrow \infty} \rho\left(T_{s}\left(x_{k}\right)-x_{k}\right)=0
$$

for every $s \in A$.

Proof Let us fix $s \in A$. Note that

$$
\rho\left(x_{j_{k}}-x_{j_{k+1}}\right) \rightarrow 0 \quad \text { as } k \rightarrow \infty
$$

By $\Delta_{2}$, it suffices to prove that

$$
\rho\left(\frac{x_{j_{k}}-x_{j_{k+1}}}{3}\right) \rightarrow 0 \quad \text { as } k \rightarrow \infty
$$


To this end observe that

$$
\rho\left(\frac{x_{j_{k}}-x_{j_{k+1}}}{3}\right) \leq \rho\left(x_{j_{k}}-x_{k}\right)+\rho\left(x_{k}-x_{k+1}\right)+\rho\left(x_{k+1}-x_{j_{k+1}}\right) \rightarrow 0,
$$

in view of the assumption (a) and by (4.9) in Lemma 4.4. Observe that

$$
\rho\left(x_{j_{k}}-T_{s}\left(x_{j_{k}}\right)\right) \rightarrow 0 \quad \text { as } k \rightarrow \infty
$$

Indeed,

$$
\begin{aligned}
\rho\left(\frac{x_{j_{k}}-T_{s}\left(x_{j_{k}}\right)}{5}\right) \leq & \rho\left(x_{j_{k}}-x_{j_{k+1}}\right)+\rho\left(x_{j_{k+1}}-T_{t_{j_{k+1}}}\left(x_{j_{k+1}}\right)\right) \\
& +\rho\left(T_{t_{j_{k+1}}}\left(x_{j_{k+1}}\right)-T_{t_{j_{k+1}}}\left(x_{j_{k}}\right)\right)+\rho\left(T_{t_{j_{k+1}}}\left(x_{j_{k}}\right)-T_{s+t_{j_{k}}}\left(x_{j_{k}}\right)\right) \\
& +\rho\left(T_{s+t_{j_{k}}}\left(x_{j_{k}}\right)-T_{s}\left(x_{j_{k}}\right)\right) \\
\leq & \rho\left(x_{j_{k}}-x_{j_{k+1}}\right)+\rho\left(x_{j_{k+1}}-T_{t_{j_{k+1}}}\left(x_{j_{k+1}}\right)\right)+a_{t_{j_{k+1}}}\left(x_{j_{k+1}}\right) \rho\left(x_{j_{k+1}}-x_{j_{k}}\right) \\
& +a_{s+t_{j_{k}}}\left(x_{j_{k}}\right) \rho\left(T_{d_{k}}\left(x_{j_{k}}\right)-x_{j_{k}}\right)+\sup _{x \in C} a_{s}(x) \rho\left(T_{t_{j_{k}}}\left(x_{j_{k}}\right)-x_{j_{k}}\right),
\end{aligned}
$$

which tends to zero as $k \rightarrow \infty$ because of (4.17), Lemma 4.4, the fact that the process is well defined, assumptions (b) and (2.9), and the boundedness of each function $a_{s}$. This convergence gives us, via $\Delta_{2}$, the required (4.20). On the other hand,

$$
\begin{aligned}
\rho\left(\frac{x_{k}-T_{s}\left(x_{k}\right)}{5}\right) \leq & \rho\left(x_{k}-x_{j_{k}}\right)+\rho\left(x_{j_{k}}-T_{t_{j_{k}}}\left(x_{j_{k}}\right)\right)+\rho\left(T_{t_{j_{k}}}\left(x_{j_{k}}\right)-T_{s+t_{j_{k}}}\left(x_{j_{k}}\right)\right) \\
& +\rho\left(T_{s+t_{j_{k}}}\left(x_{j_{k}}\right)-T_{s}\left(x_{j_{k}}\right)\right)+\rho\left(T_{s}\left(x_{j_{k}}\right)-T_{s}\left(x_{k}\right)\right) \\
\leq & \rho\left(x_{k}-x_{j_{k}}\right)+\rho\left(x_{j_{k}}-T_{t_{j_{k}}}\left(x_{j_{k}}\right)\right)+a_{t_{j_{k}}}\left(x_{j_{k}}\right) \rho\left(x_{j_{k}}-T_{s}\left(x_{j_{k}}\right)\right) \\
& +a_{s}\left(x_{j_{k}}\right) \rho\left(T_{t_{j_{k}}}\left(x_{j_{k}}\right)-x_{j_{k}}\right)+a_{s}\left(x_{k}\right) \rho\left(x_{j_{k}}-x_{k}\right),
\end{aligned}
$$

which tends to zero as $k \rightarrow \infty$ because of assumption (a), Lemma 4.4, (4.20), the fact that the process is well defined, and the fact that the semigroup is asymptotic pointwise nonexpansive. Since $\rho$ has the $\Delta_{2}$ property,

$$
\rho\left(x_{k}-T_{s}\left(x_{k}\right)\right) \rightarrow 0 \quad \text { as } k \rightarrow \infty
$$

which completes the proof of the lemma.

The following theorem is an immediate consequence of Lemma 4.5 and Theorem 4.1.

Theorem 4.2 Let $\rho \in \Re$ be uniformly continuous function modular satisfying (UUC1). Assume in addition that $\rho$ satisfies $\Delta_{2}$ and has the strong Opial property. Let $C \subset L_{\rho}$ be a $\rho$-closed, $\rho$-bounded, and convex set and let $\mathcal{F} \in \mathcal{S}(C)$. Denote by $\left\{x_{k}\right\}$ the sequence generated by a well defined generalized Krasnosel'skii-Mann process $\operatorname{gKM}\left(\mathcal{F},\left\{c_{k}\right\},\left\{t_{k}\right\}\right)$. Let $s_{1}, s_{1} \in J$ be such that $\frac{s_{1}}{s_{2}}$ is irrational and that there exists a strictly increasing sequence of natural numbers $\left\{j_{k}\right\}$ satisfying the following conditions:

(a) $\rho\left(x_{k}-x_{j_{k}}\right) \rightarrow 0$ as $k \rightarrow \infty$, 
(b) $\lim _{k \rightarrow \infty} \rho\left(T_{d_{k}}\left(x_{j_{k}}\right)-x_{j_{k}}\right)=0$, where $d_{k}=\left|t_{j_{k+1}}-t_{j_{k}}-s_{1}\right|$,

(c) $\lim _{k \rightarrow \infty} \rho\left(T_{e_{k}}\left(x_{j_{k}}\right)-x_{j_{k}}\right)=0$, where $e_{k}=\left|t_{j_{k+1}}-t_{j_{k}}-s_{2}\right|$.

Then the sequence $\left\{x_{k}\right\}$ converges $\rho$-a.e. to a common fixed point $w \in F(\mathcal{F})$.

Remark 4.1 Observe that a sequence $\left\{t_{k}\right\}$ satisfying assumptions of Theorem 4.2 can be always constructed. The main difficulty is in ensuring that the corresponding process $\operatorname{gKM}\left(\mathcal{F},\left\{c_{k}\right\},\left\{t_{k}\right\}\right)$ is well defined.

The next result answers the question when the sequence generated by the generalized Krasnosel'skii-Mann process will converge strongly to a common fixed point. Not surprisingly we need to add a compactness assumption.

Theorem 4.3 Under the assumptions of Theorem 4.2, if in addition $C$ is assumed to be $\rho$-compact, then the sequence $\left\{x_{k}\right\}$ generated by $\operatorname{gKM}\left(\mathcal{F},\left\{c_{k}\right\},\left\{t_{k}\right\}\right)$ converges strongly to a common fixed point $w \in F(\mathcal{F})$, that is,

$$
\lim _{k \rightarrow \infty} \rho\left(x_{k}-w\right)=0
$$

Proof It follows from Theorem 4.2 that there exists a common fixed point $w \in F(\mathcal{F})$ such that $\left\{x_{k}\right\}$ converges $\rho$-a.e. By $\rho$-compactness of $C$ there exist $x \in C$ and a subsequence $\left\{x_{p_{k}}\right\}$ of $\left\{x_{k}\right\}$ such that

$$
\lim _{k \rightarrow \infty} \rho\left(x_{p_{k}}-x\right)=0
$$

By Proposition 2.1 there exists a subsequence $\left\{x_{p_{k_{n}}}\right\}$ of $\left\{x_{p_{k}}\right\}$ such that

$$
x_{p_{k}} \rightarrow x \quad \rho \text {-a.e. }
$$

By the uniqueness of the $\rho$-a.e. limit we get $x=w$. Hence

$$
\lim _{k \rightarrow \infty} \rho\left(x_{p_{k}}-w\right)=0
$$

On the other hand, the limit $\lim _{k \rightarrow \infty} \rho\left(x_{k}-w\right)$ exists by Lemma 4.3, which implies that

$$
\lim _{k \rightarrow \infty} \rho\left(x_{k}-w\right)=0
$$

as claimed.

Remark 4.2 Observe that in view of the $\Delta_{2}$ assumption, the $\rho$-compactness of the set $C$ assumed in Theorem 4.3 is equivalent to the compactness in the sense of the norm defined by $\rho$.

Competing interests

The authors declare that they have no competing interests. 


\section{Author details}

${ }^{1}$ Department of Mathematics, King Abdulaziz University, P.O. Box 53909, Jeddah, 21593, Saudi Arabia. ${ }^{2}$ Department of Mathematical Sciences, University of Texas at El Paso, El Paso, TX, USA. ${ }^{3}$ Department of Mathematics and Statistics, King Fahd University of Petroleum and Minerals, Dhahran, 31261, Saudi Arabia. ${ }^{4}$ School of Mathematics and Statistics, University of New South Wales, Sydney, NSW 2052, Australia.

\section{Acknowledgements}

This work was funded by the Deanship of Scientific Research (DSR), King Abdulaziz University, Jeddah, under Grant No. (247-001-D1434). The authors, therefore, acknowledge with thanks technical and financial support of DSR.

\section{Received: 9 August 2014 Accepted: 8 December 2014 Published: 16 Jan 2015}

\section{References}

1. Kirk, WA, Xu, HK: Asymptotic pointwise contractions. Nonlinear Anal. 69, 4706-4712 (2008)

2. Hussain, N, Khamsi, MA: On asymptotic pointwise contractions in metric spaces. Nonlinear Anal. 71(10), 4423-4429 (2009)

3. Khamsi, MA, Kozlowski, WM: On asymptotic pointwise nonexpansive mappings in modular function spaces. J. Math. Anal. Appl. 380(2), 697-708 (2011)

4. Kozlowski, WM: On the existence of common fixed points for semigroups of nonlinear mappings in modular function spaces. Comment. Math. 51(1), 81-98 (2011)

5. Bin Dehaish, BA, Khamsi, MA, Kozlowski, WM: Common fixed points for asymptotic pointwise Lipschitzian semigroups in modular function spaces. Fixed Point Theory Appl. 2013, 214 (2013)

6. Bin Dehaish, BA, Kozlowski, WM: Fixed point iterations processes for asymptotic pointwise nonexpansive mappings in modular function spaces. Fixed Point Theory Appl. 2012, 118 (2012)

7. Kozlowski, WM: Modular Function Spaces. Series of Monographs and Textbooks in Pure and Applied Mathematics, vol. 122. Dekker, New York (1988)

8. Khamsi, MA, Kozlowski, WM, Reich, S: Fixed point theory in modular function spaces. Nonlinear Anal. 14, 935-953 (1990)

9. Khamsi, MA, Kozlowski, WM, Shutao, C: Some geometrical properties and fixed point theorems in Orlicz spaces. J. Math. Anal. Appl. 155(2), 393-412 (1991)

10. Khamsi, MA: Nonlinear semigroups in modular function spaces. Math. Jpn. 37(2), 1-9 (1992)

11. Khamsi, MA: Fixed point theory in modular function spaces. In: Proceedings of the Workshop on Recent Advances on Metric Fixed Point Theory (Seville, September 1995), pp. 31-57 (1996)

12. Dominguez-Benavides, T, Khamsi, MA, Samadi, S: Uniformly Lipschitzian mappings in modular function spaces. Nonlinear Anal. 46, 267-278 (2001)

13. Dominguez-Benavides, T, Khamsi, MA, Samadi, S: Asymptotically regular mappings in modular function spaces. Sci. Math. Jpn. 53, 295-304 (2001)

14. Dominguez-Benavides, T, Khamsi, MA, Samadi, S: Asymptotically nonexpansive mappings in modular function spaces. J. Math. Anal. Appl. 265(2), 249-263 (2002)

15. Hajji, A, Hanebaly, E: Perturbed integral equations in modular function spaces. Electron. J. Qual. Theory Differ. Equ. 2003, 20 (2003). http://www.math.u-szeged.hu/ejqtde/

16. Khamsi, MA, Kozlowski, WM: On asymptotic pointwise contractions in modular function spaces. Nonlinear Anal. 73 , 2957-2967 (2010)

17. Kozlowski, WM: Advancements in fixed point theory in modular function. Arab. J. Math. (2012). doi:10.1007/s40065-012-0051-0

18. Kozlowski, WM: An introduction to fixed point theory in modular function spaces. In: Almezel, S, Ansari, QH, Khamsi, MA (eds.) Topics in Fixed Point Theory. Springer, Cham (2014)

19. Kozlowski, WM: On nonlinear differential equations in generalized Musielak-Orlicz spaces. Comment. Math. 53(2), 113-133 (2013)

20. Kozlowski, WM: On the Cauchy problem for the nonlinear differential equations with values in modular function spaces. In: Proceeding of International Conference in Differential Geometry, Functional Analysis and Applications (8-10 September 2012, Jamia Millia Islamia University, New Delhi, India) Narosa Publicactions, New Delhi (2014)

21. Bose, SC: Weak convergence to the fixed point of an asymptotically nonexpansive. Proc. Am. Math. Soc. 68, 305-308 (1978)

22. Samanta, SK: Fixed point theorems in a Banach space satisfying Opial's condition. J. Indian Math. Soc. 45, 251-258 (1981)

23. Passty, GB: Construction of fixed points for asymptotically nonexpansive mappings. Proc. Am. Math. Soc. 84, 212-216 (1982)

24. Gornicki, J: Weak convergence theorems for asymptotically nonexpansive mappings in uniformly convex Banach spaces. Comment. Math. Univ. Carol. 30, 249-252 (1989)

25. Schu, J: Iterative construction of fixed points of asymptotically nonexpansive mappings. J. Math. Anal. Appl. 158, 407-413 (1991)

26. Schu, J: Weak and strong convergence to fixed points of asymptotically nonexpansive mappings. Bull. Aust. Math Soc. 43, 153-159 (1991)

27. Tan, K-K, Xu, H-K: The nonlinear ergodic theorem for asymptotically nonexpansive mappings in Banach spaces. Proc Am. Math. Soc. 114, 399-404 (1992)

28. $\mathrm{Xu}, \mathrm{H}-\mathrm{K}$ : Existence and convergence for fixed points of asymptotically nonexpansive type. Nonlinear Anal. 16 1139-1146 (1991)

29. Tan, K-K, Xu, H-K: A nonlinear ergodic theorem for asymptotically nonexpansive mappings. Bull. Aust. Math. Soc. 45 25-36 (1992)

30. Tan, K-K, Xu, H-K: Approximating fixed points of nonexpansive mappings by the Ishikawa iteration process. J. Math. Anal. Appl. 178, 301-308 (1993) 
31. Bruck, R, Kuczumow, T, Reich, S: Convergence of iterates of asymptotically nonexpansive mappings in Banach spaces with the uniform Opial property. Colloq. Math. 65(2), 169-179 (1993)

32. Tan, K-K, Xu, H-K: Fixed point iteration processes for asymptotically nonexpansive mappings. Proc. Am. Math. Soc. $122,733-739(1994)$

33. Rhoades, BE: Fixed point iterations for certain nonlinear mappings. J. Math. Anal. Appl. 183, 118-120 (1994)

34. Noor, MA, Xu, B: Fixed point iterations for asymptotically nonexpansive mappings in Banach spaces. J. Math. Anal. Appl. 267, 444-453 (2002)

35. Khamsi, MA: On asymptotically nonexpansive mappings in hyperconvex metric spaces. Proc. Am. Math. Soc. 132, 365-373 (2004)

36. Fukhar-ud-din, $H$, Khan, AR: Approximating common fixed points of asymptotically nonexpansive maps in uniformly convex Banach spaces. Comput. Math. Appl. 53, 1349-1360 (2009)

37. Nanjaras, B, Panyanak, B: Demiclosed principle for asymptotically nonexpansive mappings in CAT(0) spaces. Fixed Point Theory Appl. 2010, Article ID 268780 (2010)

38. Kozlowski, WM: Fixed point iteration processes for asymptotic pointwise nonexpansive mappings in Banach spaces. J. Math. Anal. Appl. 377(1), 43-52 (2011)

39. Kozlowski, WM: Common fixed points for semigroups of pointwise Lipschitzian mappings in Banach spaces. Bull. Aust. Math. Soc. 84, 353-361 (2011)

40. Kozlowski, WM, Sims, B: On the convergence of iteration processes for semigroups of nonlinear mappings in Banach spaces. In: Bailey, DH, Bauschke, HH, Borwein, P, Garvan, F, Thera, M, Vanderwerff, JD, Wolkowicz, H (eds.) Computational and Analytical Mathematics: In Honor of Jonathan Borwein's 60th Birthday. Springer Proceedings in Mathematics and Statistics, vol. 50. Springer, New York (2013)

41. Kozlowski, WM: On the construction of common fixed points for semigroups of nonlinear mappings in uniformly convex and uniformly smooth Banach spaces. Comment. Math. 52(2), 113-136 (2012)

42. Kozlowski, WM: Notes on modular function spaces I. Comment. Math. 28, 91-104 (1988)

43. Kozlowski, WM: Notes on modular function spaces II. Comment. Math. 28, 105-120 (1988)

44. Chen, S: Geometry of Orlicz spaces. Diss. Math. 356, 1-204 (1996)

45. Kaminska, A: On uniform convexity of Orlicz spaces. Indag. Math. 44(1), 27-36 (1982)

46. Khamsi, MA: A convexity property in modular function spaces. Math. Jpn. 44(2), 269-279 (1996)

47. Stewart, I, Tall, D: Algebraic Number Theory and Fermat's Last Theorem, 3rd edn. AK Peters, Natick (2001)

10.1186/1687-1812-2015-3

Cite this article as: Bin Dehaish et al.: On the convergence of iteration processes for semigroups of nonlinear mappings in modular function spaces. Fixed Point Theory and Applications 2015, 2015:3

\section{Submit your manuscript to a SpringerOpen ${ }^{\circ}$ journal and benefit from:}

- Convenient online submission

- Rigorous peer review

- Immediate publication on acceptance

- Open access: articles freely available online

- High visibility within the field

- Retaining the copyright to your article 